Correspondence

Ambrose Y. Jong

ajong@chla.usc.edu

Received 20 February 2003

Accepted 3 July 2003

\section{Cryptococcus neoformans induces alterations in the cytoskeleton of human brain microvascular endothelial cells}

\author{
Steven H. M. Chen, ${ }^{1}$ Monique F. Stins, ${ }^{2}$ Sheng-He Huang, ${ }^{3}$ Yu Hua Chen, ${ }^{3}$ \\ K. J. Kwon-Chung, ${ }^{4}$ Yun Chang, ${ }^{4}$ Kwang Sik Kim, ${ }^{2}$ Kazuhiro Suzuki ${ }^{5}$ \\ and Ambrose Y. Jong ${ }^{1}$ \\ 1,3 Divisions of Hematology-Oncology ${ }^{1}$ and Infectious Diseases ${ }^{3}$, Children's Hospital Los Angeles, \\ Los Angeles, CA 90027, USA \\ ${ }^{2}$ The Johns Hopkins University, Pediatric Infectious Diseases, 600 N. Wolfe St, Park 256, Baltimore, \\ MD 21287, USA \\ ${ }^{4}$ Laboratory of Clinical Investigation, National Institute of Allergy and Infectious Diseases, Bethesda, \\ MD 20892, USA \\ ${ }^{5}$ National Institute of Health Sciences, Tokyo, Japan
}

The fungal pathogen Cryptococcus neoformans has a predilection for the central nervous system (CNS), resulting in devastating meningoencephalitis. At present, it is unclear how C. neoformans traverses the blood-brain barrier (BBB) and causes CNS infection. The present study has examined and characterized the interaction of $C$. neoformans with human brain microvascular endothelial cells (HBMEC), which constitute the BBB. Adhesion of and transcytosis of HBMEC by C. neoformans was inoculum- and time-dependent and occurred with both encapsulated and acapsulated strains. C. neoformans induced marked morphological changes in HBMEC, for example membrane ruffling, irregular nuclear morphology and swelling of the mitochondria and the ER. These findings suggest that C. neoformans induced actin cytoskeletal reorganization of the host cells. In addition, it was observed that the dephosphorylated form of cofilin was increased during cryptococcal adherence to HBMEC, concomitant with the actin rearrangement. Cryptococcal binding to HBMEC was increased in the presence of Y27632, a Rho kinase (ROCK)-specific inhibitor. Since ROCK activates LIM kinase (LIMK), which phosphorylates cofilin (inactive form), this suggests the involvement of the ROCK $\rightarrow$ LIMK $\rightarrow$ cofilin pathway. In contrast, the phosphatase inhibitor sodium orthovanadate decreased adherence of Cryptococcus to HBMEC, concomitant with the increase of phosphorylation of cofilin. Furthermore, the tight junction marker protein occludin became Tritonextractable, indicating alteration of tight junctions in brain endothelial cells. This is the first demonstration that C. neoformans is able to adhere to and transcytose across the HBMEC monolayer and alter the cytoskeleton morphology in HBMEC. Further characterization of the interactions between C. neoformans and HBMEC should help the development of novel strategies to prevent cryptococcal meningitis and its associated morbidity.

\section{INTRODUCTION}

Infection by Cryptococcus neoformans, an encapsulated fungal organism, has increased considerably over recent years (Mitchell \& Perfect, 1995; Gottfredsson \& Perfect, 2000; Kwon-Chung et al., 2000). The expanded use of immuno-

Abbreviations: BBB, blood-brain barrier; CNS, central nervous system; HBMEC, human brain microvascular endothelial cell; HUVEC, human umbilical vein endothelial cell; LIMK, LIM kinase; ROCK, Rho kinase; TEER, trans-endothelial electrical resistance. suppressive drugs and the onset of AIDS have contributed to this epidemic. Patients with T-cell deficiencies, including AIDS, lymphoma, corticosteroid therapy and idiopathic CD4 lymphocytopenia, are the most susceptible to C. neoformans infection. Dehydrated haploid yeast or basidiospores of C. neoformans enter the host via the respiratory system. They are then likely to spread haematogeneously to extrapulmonary tissues, including the brain, where meningoencephalitis develops. Untreated cryptococcal meningoencephalitis is fatal in normal hosts. Even when treated with the most 
effective antifungal drugs, C. neoformans meningoencephalitis causes high mortality and morbidity in patients without adequate $\mathrm{T}$-cell-dependent immune function. In order to cause meningitis, C. neoformans must penetrate the bloodbrain barrier (BBB). The BBB is a barrier between blood circulation and the brain parenchyma and consists mainly of specialized capillary endothelium, characterized, for example, by the presence of tight junctions. It is responsible for maintaining biochemical homeostasis within the central nervous system (CNS) (Broadwell et al., 1996; Rubin \& Staddon, 1999). In general, microbial pathogens may breach the $\mathrm{BBB}$ and enter the CNS through transcellular penetration, paracellular entry and/or transmigration with infected leucocytes (Huang \& Jong, 2001). The underlying mechanism(s) whereby $C$. neoformans crosses the $\mathrm{BBB}$ is unclear.

It is commonly observed that the host-cell actin cytoskeleton undergoes extensive remodelling during pathogenic invasion (Galan \& Zhou, 2000; Masuda et al., 2000; Chen et al., 2002; Eugene et al., 2002; Khan et al., 2002). The small GTPase Rho regulates such remodelling, but the underlying mechanisms of this regulation vary depending on the pathogen (Kim, 2001). Despite differences in mechanisms of invasion, various activated host signals are transduced downstream, converging within the actin machinery, and result in cytoskeletal reorganization. Actin dynamics are tightly regulated at multiple levels. Recent studies have indicated that phosphorylation regulation of cofilin plays an important role in actin dynamics (Bamburg, 1999; Bierne et al., 2001). Cofilin is phosphorylated by LIM kinase (LIMK), which inhibits cofilin function and stabilizes actin reorganization (Arber et al., 1998; Yang et al., 1998; Sumi et al., 1999). The dephosphorylated cofilin promotes actin depolymerization at the pointed end, prompting actin reorganization. Dephosphorylation of cofilin by phosphatase (Niwa et al., 2002) stimulates its activity and actin reorganization. Little is known at present about fungus-induced host-cell cytoskeletal modifications.

To date, much effort has been devoted to identifying and characterizing virulence factors involved in the pathogenicity of $C$. neoformans. The dominant virulence factors are the polysaccharide capsular components and soluble extracellular constituents (Kwon-Chung \& Rhodes, 1986; Chang \& Kwon-Chung, 1994). Capsular components of C. neoformans such as mannoproteins have been shown to interact with the immune system. They are also likely to interact with susceptible host cells. Thus, they may play an important role in pathogenesis (Hamilton \& Goodley, 1996; Buchanan \& Murphy, 1998). Recent gene disruption and complementation studies in a mouse model have provided unambiguous genetic evidence to demonstrate that the capsular genes CAP10, CAP59, CAP60 and CAP64 of C. neoformans are virulent (Chang \& Kwon-Chung, 1994, 1998, 1999; Chang et al., 1996). Several other factors, including melanin synthesis, MAT $\alpha$, phospholipases and thermotolerance at $37^{\circ} \mathrm{C}$, have been shown to be associated with virulence (Hamilton \& Goodley, 1996; Hogan et al., 1996; Buchanan \& Murphy, 1998; Perfect et al., 1998).
Most currently identified cryptococcal virulence factors are involved in the survival of pathogens in the host system, but microbial toxin-like compounds or invasive proteins have yet to be demonstrated. Although interactions between $C$. neoformans and immune cells (Feldmesser et al., 2000) and human umbilical vein endothelial cells (HUVEC) (Ibrahim et al., 1995) have been described, interaction of C. neoformans with human brain microvascular endothelial cells (HBMEC) has not yet been investigated, particularly as it pertains to the ability to cross the BBB. In this report, we have examined the interaction of C. neoformans with HBMEC by using in vitro adhesion and transcytosis assays in combination with transmission electron microscopic and confocal microscopic methods. We show that $C$. neoformans induces considerable morphological changes and actin reorganization in HBMEC through the phosphorylation regulation of cofilin and related pathways. Taken together, these alterations, at the cellular and molecular levels, indicate unique characteristics of HBMEC responses during Cryptococcus invasion.

\section{METHODS}

Yeast strains. C. neoformans strains B3501, 602, TYCC38-602, TYCC33 and B4476 FO5 have been described previously (Chang et al., 1996; Chang \& Kwon-Chung, 1998, 1999). Briefly, strain B3501 is a highly encapsulated strain, strain 602 is a cap64 mutant strain, strain TYCC38-602 is an encapsulated transformant of 602 with wild-type CAP64 gene and strain TYCC33 was generated by gene deletion of CAP59 and is an isogenic strain of B4476 FO5 (CAP59). Yeast cells were grown aerobically at $30{ }^{\circ} \mathrm{C}$ in $1 \%$ yeast extract, $2 \%$ peptone and $2 \%$ glucose (YPD broth) or Sabouraud medium (Difco). Cells were harvested at early exponential phase, washed with PBS and resuspended in Hams-F12/M199 (1:1, v/v), $5 \%$ heat inactivated fetal bovine serum (FBS) (experimental medium) and $1 \%$ fresh human serum. The number of Cryptococcus cells was determined by direct counting from a haemocytometer, as spectrophotometric readings were found to be inaccurate because of clumping of the organism, especially the acapsular strains.

Isolation, characterization and culture of HBMEC. HBMEC were isolated from human brain specimens obtained post mortem or by surgical resection during surgery for seizure disorder and characterized as described previously (Stins et al., 1997a, b). Briefly, brain specimens were cut into small pieces and homogenized in DMEM containing $2 \%$ FBS (DMEM-S) using a Dounce homogenizer with a loose fitting. The homogenate was centrifuged in $15 \%$ dextran in DMEM-S for $10 \mathrm{~min}$ at $10000 \mathrm{~g}$. The pellet containing crude microvessels was further digested in a solution containing $1 \mathrm{mg}$ collagenase/dispase $\mathrm{ml}^{-1}$ in DMEM-S for $1 \mathrm{~h}$ at $37^{\circ} \mathrm{C}$. Microvascular capillaries were isolated by absorption to a column of glass beads $(0 \cdot 25-0 \cdot 3 \mathrm{~mm})$ and washing from the beads. HBMEC were plated on rat tail collagen/fibronectin-coated dishes or glass coverslips and cultured in RPMI 1640-based medium with growth factors, $10 \%$ heat-inactivated FBS, $10 \%$ NuSerum, $5 \mathrm{U}_{\text {heparin }} \mathrm{ml}^{-1}, 2$ mM L-glutamine, $1 \mathrm{mM}$ sodium pyruvate, non-essential amino acids, vitamins and $100 \mathrm{U}$ penicillin and streptomycin $\mathrm{ml}^{-1}$. Viability of HBMEC was assessed by examining cellular morphology and by trypan blue exclusion. HBMEC were positive for factor VIII-Rag, took up fluorescently labelled acetylated low-density lipoprotein and expressed $\gamma$-glutamyl transpeptidase, thus demonstrating their brain endothelial cell characteristics. HBMEC cultures were maintained in RPMI-based medium, including $10 \%$ FBS and $10 \%$ NuSerum (BD Biosciences), at $37^{\circ} \mathrm{C}$ in a humid atmosphere of $5 \% \mathrm{CO}_{2}$ as described previously (Nizet et al., 1997). 
Adhesion of $\boldsymbol{C}$. neoformans in HBMEC. Total HBMEC-associated yeast was determined as described previously (Jong et al., 2001) with the following modifications. HBMEC were grown in collagen-coated 24well tissue-culture plates (Costar) until confluence. An inoculum of $10^{4}$, $10^{5}, 10^{6}$ or $10^{7}$ Cryptococcus cells in $0.5 \mathrm{ml}$ experimental medium was added (m.o.i. of $10-10^{4}$ ) for $2 \mathrm{~h}$ at $37^{\circ} \mathrm{C}$ for titration studies or an inoculum of $10^{6}$ yeast cells per well was used (m.o.i. $\sim 10$ ) for timecourses in strain-dependent experiments. Free yeast cells were removed from HBMEC by washing four times with experimental medium. Adherent/invasive yeast cells were retained by the HBMEC. Subsequently, HBMEC were lysed with $0.5 \%$ Triton, diluted and plated onto blood or YPD agar plates and colonies were counted. For time-course studies, the incubated cultures were harvested at indicated time-points for colony counting. Viability of HBMEC was assessed as described above. Results are presented as either the total number of colonies or the percentage adhesion of inoculum [100\% $\times$ (number of Cryptococcus recovered)/(number of Cryptococcus inoculated)]. In some experiments, the inhibitors Y27632, sodium orthovanadate or okadaic acid (Sigma) were used. These inhibitors were added to HBMEC $1 \mathrm{~h}$ before the addition of $C$. neoformans. They were washed away with experimental medium before the addition of Cryptococcus cells.

Transcytosis. HBMEC were cultured on collagen-coated Transwell polycarbonate tissue-culture inserts with a pore diameter of $12 \mu \mathrm{m}$ (Corning Costar) for 5 days (Jong et al., 2001). HBMEC were polarized and exhibited a trans-endothelial electrical resistance (TEER) of $200-250 \Omega \mathrm{cm}^{-2}$, as measured with an Endohm volt/ohm meter in conjunction with an Endohm chamber (World Precision Instruments) as described previously (Jong et al., 2001). On the morning of the assay, HBMEC monolayers were washed with experimental medium and $10^{6}$ Cryptococcus cells were added to the upper chamber (total volume $200 \mu \mathrm{l})$, and were then incubated at $37^{\circ} \mathrm{C}$. At $0,3,6,12,24$ and $48 \mathrm{~h}$, samples $(100 \mu \mathrm{l})$ were taken from the lower chamber (an equivalent volume of medium was immediately replaced to maintain a total volume of $1 \mathrm{ml}$ in the lower chamber) and plated for counting of c.f.u. Simultaneously, the integrity of the HBMEC monolayer was assessed by measurement of the TEER. Three measurements were made at each time-point for each sample.

Transmission electron microscopy (TEM). TEM was used to evaluate the effect of Cryptococcus on HBMEC at the ultrastructural level. HBMEC were incubated with Cryptococcus for $20 \mathrm{~h}$ at $37^{\circ} \mathrm{C}$, washed four times with experimental medium, fixed with $4 \%$ paraformaldehyde $/ 2.5 \%$ glutaraldehyde in PBS for at least 10 min at $4{ }^{\circ} \mathrm{C}$ and carefully scraped from their support. The cell slurry was then centrifuged for $10 \mathrm{~min}$ at $7000 \mathrm{~g}$ and processed for TEM. Briefly, the HBMEC pellet was post-fixed with buffered $2 \% \mathrm{OsO}_{4}$ for $1 \mathrm{~h}$, dehydrated through graded ethanol solutions and propylene oxide and embedded in Epon. Ultrathin sections were cut, stained with uranyl acetate and lead citrate and examined with a Philips CM TEM.

Confocal microscopy. Samples for phase-contrast microscopy were prepared as follows. HBMEC were plated onto glass coverslips $(22 \mathrm{~mm}$, square), which had previously been coated with fibronectin $\left(5 \mathrm{mg} \mathrm{ml}^{-1}\right)$ or polylysine $\left(5 \mathrm{mg} \mathrm{ml}^{-1}\right)$, in an 8 -well square culture plate (Nalgen Nunc). HBMEC $\left(5 \times 10^{4}\right)$ were seeded onto a coverslip $24 \mathrm{~h}$ before the experiment. Treated HBMEC were prewashed four times with PBS and then fixed with $4 \%$ formaldehyde in PBS for 20 min at room temperature. After an additional three washes with PBS, the HBMEC were permeabilized by $0.2 \%$ Triton X-100 in PBS for 2 min. The coverslips were then washed extensively three times with PBS and blocked with $1 \%$ BSA in PBS for $30 \mathrm{~min}$. Rhodamine-labelled phalloidin $\left(1 \mu \mathrm{g} \mathrm{m} \mathrm{m}^{-1}\right)$ (Sigma) in PBS plus 1\% BSA was added to each well and the coverslip was incubated for $1 \mathrm{~h}$ at room temperature. Another three washes were applied before sealing the coverslips onto slides. Samples were examined with a Leica TCS SP confocal DM IRBE inverted fluorescence micro- scope equipped with PlanApochromat 40×/1.25NA oil-immersion lenses (CHLA Congressman Dixon Cellular Imaging Core Facility).

Protein blots. Protein concentration was determined by Bio-Rad protein assay. Equal amounts of protein sample were used. SDS-PAGE sample buffer (50 mM Tris/ $\mathrm{HCl}, \mathrm{pH} 6 \cdot 8,10 \% \beta$-mercaptoethanol, $2 \%$ SDS, $0 \cdot 1 \%$ bromophenol blue, $10 \%$ glycerol) was added to the samples and they were then boiled in a water bath for $10 \mathrm{~min}$. Lysate was stored at $-20{ }^{\circ} \mathrm{C}$ for later analysis. Proteins were separated on homogeneous $12.5 \%$ Phast gel (Phast System, Amersham Biosciences) with SDS buffer strips according to the manufacturer's instructions with subsequent transfer to PVDF membrane. The membrane was blocked with $0.5 \%$ blocking solution (skimmed milk-based) and incubated with antibodies prepared against cofilin, phospho-cofilin (p-cofilin), occludin (Transduction Laboratories) or actin (Chemicon International) at room temperature. This was followed by incubation with peroxidase-coupled secondary antibody (Kirkegaard Perry Laboratories). The blots were examined with the ECL enhanced chemiluminescent system (Boehringer Mannheim).

Statistical analysis. Data are expressed as means \pm SE of at least three repeat experiments. Analysis of variance (ANOVA) followed by the Newmann-Keuls test was used to determine the statistical significance between the control and treatment groups. $P<0.05$ was considered to be significant.

\section{RESULTS}

\section{Adhesion of C. neoformans to HBMEC}

In order to penetrate the CNS, Cryptococcus cells must adhere to and cross the endothelium of the BBB. We initially investigated the ability of $C$. neoformans strains to adhere to HBMEC in vitro. Adhesion of C. neoformans strain B3501 to HBMEC was time-dependent, showing linear adhesion up to $4 \mathrm{~h}$ with $10^{6}$ yeast cells $\mathrm{ml}^{-1}$. The effect of inoculum size on binding of $C$. neoformans B3501 to HBMEC was also evaluated. With increasing inoculum from $10^{4}$ to $10^{7} \mathrm{ml}^{-1}$, a concomitant increase in Cryptococcus binding to HBMEC was observed. Thereafter, there appeared to be a plateau in Cryptococcus binding to HBMEC. The results showed that C. neoformans B3501 was able to adhere to HBMEC in a time- and concentration-dependent manner.

Since the capsule is known to be a virulence factor and is most likely preserved in infected patients, we examined and compared different capsulated and acapsulated Cryptococcus strains for their relative ability to adhere to HBMEC (Fig. 1). Isogenic capsulated and acapsulated strain pairs (CAP64 versus cap64 and CAP59 versus cap59) showed slightly different adhesion abilities, with the capsular stains having a slightly greater adhesion activity.

\section{Transcytosis of $C$. neoformans through the HBMEC monolayer}

Compared with bacterial and viral pathogens, C. neoformans cells are relatively large $(5-10 \mu \mathrm{m}$ diameter). How cells of this size pass through the BBB is currently unknown. We used a transcytosis assay to examine the ability of $C$. neoformans to traverse the HBMEC monolayer. Since the yeast does not divide well in the culture medium $\left(t_{1 / 2} \sim 20 \mathrm{~h}\right)$, the presence 


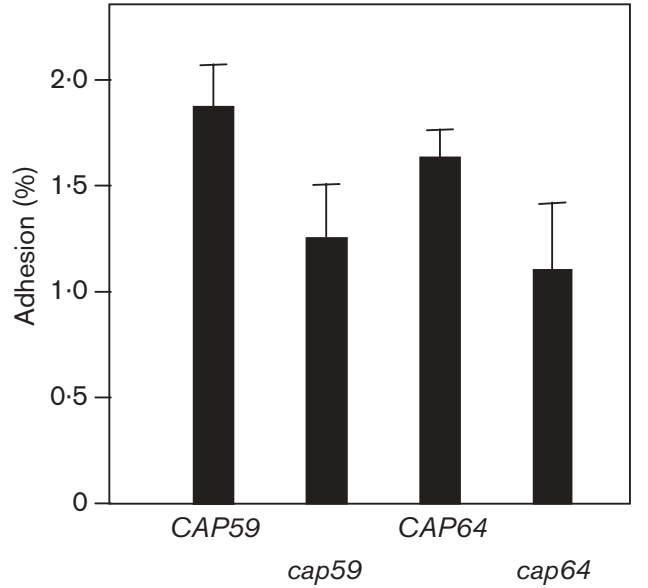

Fig. 1. Association of isogenic acapsular and capsular Cryptococcus strains with HBMEC. HBMEC were incubated with Cryptococcus strains for $1 \mathrm{~h}$ for the adhesion assay. Isogenic wild-type CAP59 (B4476) versus mutant cap59 (TYCC33) and isogenic wild-type CAP64 (TYCC38-602) versus mutant cap64 (602) are shown. Viable colonies of yeast associated with HBMEC were determined. Data, expressed as percentages of inoculum, are means \pm SE of triplicates.

of Cryptococcus cells in the lower chamber was most likely due to passage of Cryptococcus cells across the HBMEC monolayer from the upper chamber. As early as $3 \mathrm{~h}$ into the incubation, the capsulated strains B4476 (CAP59) and TYCC38-602 (CAP64) were able to pass through the HBMEC monolayer, and isogenic cap mutants could also be detected after $6 \mathrm{~h}$ incubation (Fig. 2). Thereafter, the number of c.f.u. increased with time. The capsular strains B4476 (CAP59) and TYCC38-602 (CAP64) showed a much greater ability to cross the HBMEC monolayer. Strain B3501 passed through the monolayer after $24 \mathrm{~h}$. This strain is bulky due to its heavy encapsulation. This may have affected its ability to pass through the HBMEC monolayer. There was no significant change in TEER up to $48 \mathrm{~h}$. In summary, the cell association assay and transcytosis studies indicated that $C$. neoformans, despite its size and bulky capsule, is able to adhere to and pass through the HBMEC monolayer.

\section{Morphological alteration of HBMEC by C. neoformans determined by TEM}

The effect of Cryptococcus on the HBMEC monolayer was evaluated morphologically at the ultrastructural level by TEM. In an uninfected control, HBMEC displayed their regular flat and elongated endothelial morphology with an oval-shaped nucleus (Fig. 3a). In TEM preparations of scraped endothelial cells, it was difficult to distinguish decisively between the basolateral and apical sides of the endothelial cells. The basolateral side of the endothelial cells, however, was generally flatter, and remnants of the matrix could be observed; whereas the apical side bulged slightly outwards, and the membrane was smooth and round (Fig. 3a). Intracellular organelles such as mitochondria, Golgi complex, ER and nucleus were easily recognized. When

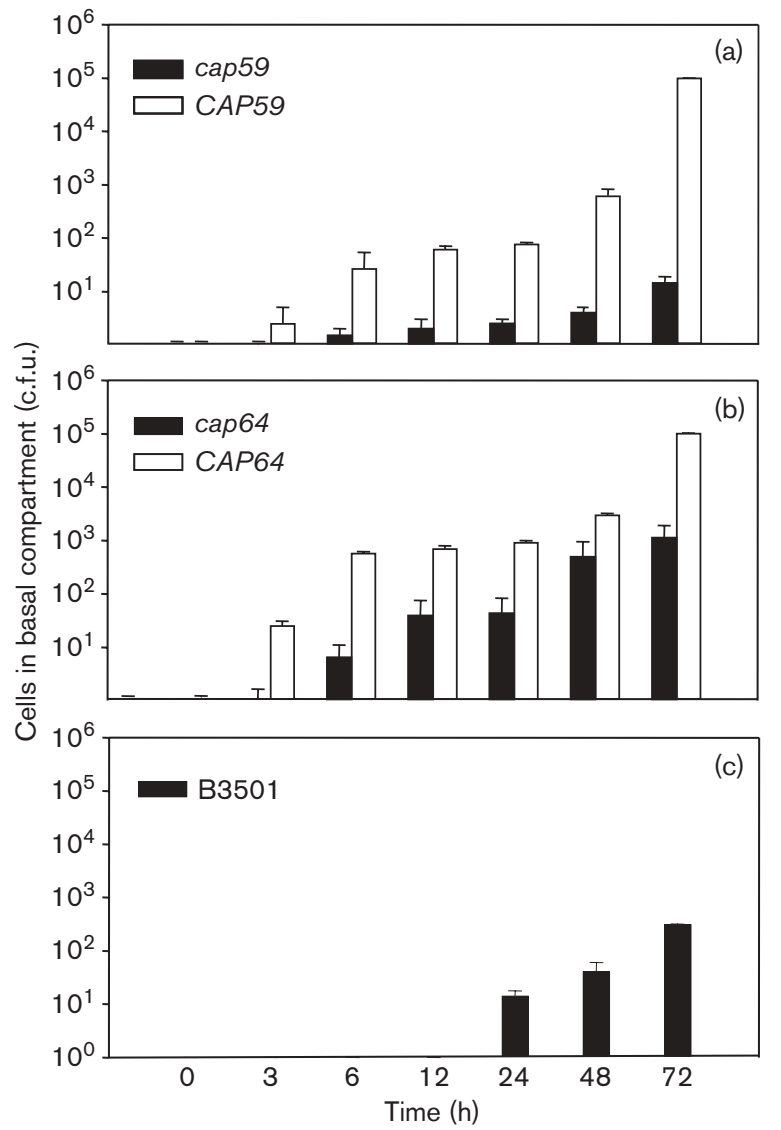

Fig. 2. Transcytosis of $C$. neoformans across an in vitro model of the BBB. Cryptococcus cells were examined in crossing Transwell filters (12 $\mu \mathrm{m}$ pore size). HBMEC formed a tight monolayer with high TEER (200-250 $\Omega$ ) as a distinct barrier to diffusion. Cryptococcus cells that crossed the Transwell filters were collected from the lateral chamber at the times indicated. Isogenic wild-type CAP59(B4476) and mutant cap59 (TYCC33) strains (a), isogenic wild-type CAP64 (TYCC38602) and mutant cap64 (602) strains (b) and strain B3501 (c) were used. Data are means \pm SE of triplicates. All the differences in the figure are significant $[P<0.05$ when compared to the $0 \mathrm{~h}$ group (ANOVA followed by Newmann-Keuls test)].

HBMEC were incubated with Cryptococcus cells, their morphology changed dramatically (Fig. 3b, c). HBMEC in these preparations appeared to be swollen, with the apical side bulging up, the apical membrane ruffling and with substantial microvilli formations. The microvilli resembled filopodial protrusions and/or apoptotic bodies. The basolateral side generally remained flat. Intracellularly, the nuclear shape was irregular. The mitochondria and ER appeared swollen and their membranes seemed detached. The alteration of cellular ultrastructure suggested that cytoskeletal rearrangement was induced during the incubation of HBMEC with Cryptococcus cells. Interestingly, despite the dramatic morphological and intracellular changes, attachment between individual endothelial cells remained intact (Fig. 3c, d; arrows). Compared with Fig. 3(c), the cell-cell attachment in Fig. 3(d) seems less contiguous, 

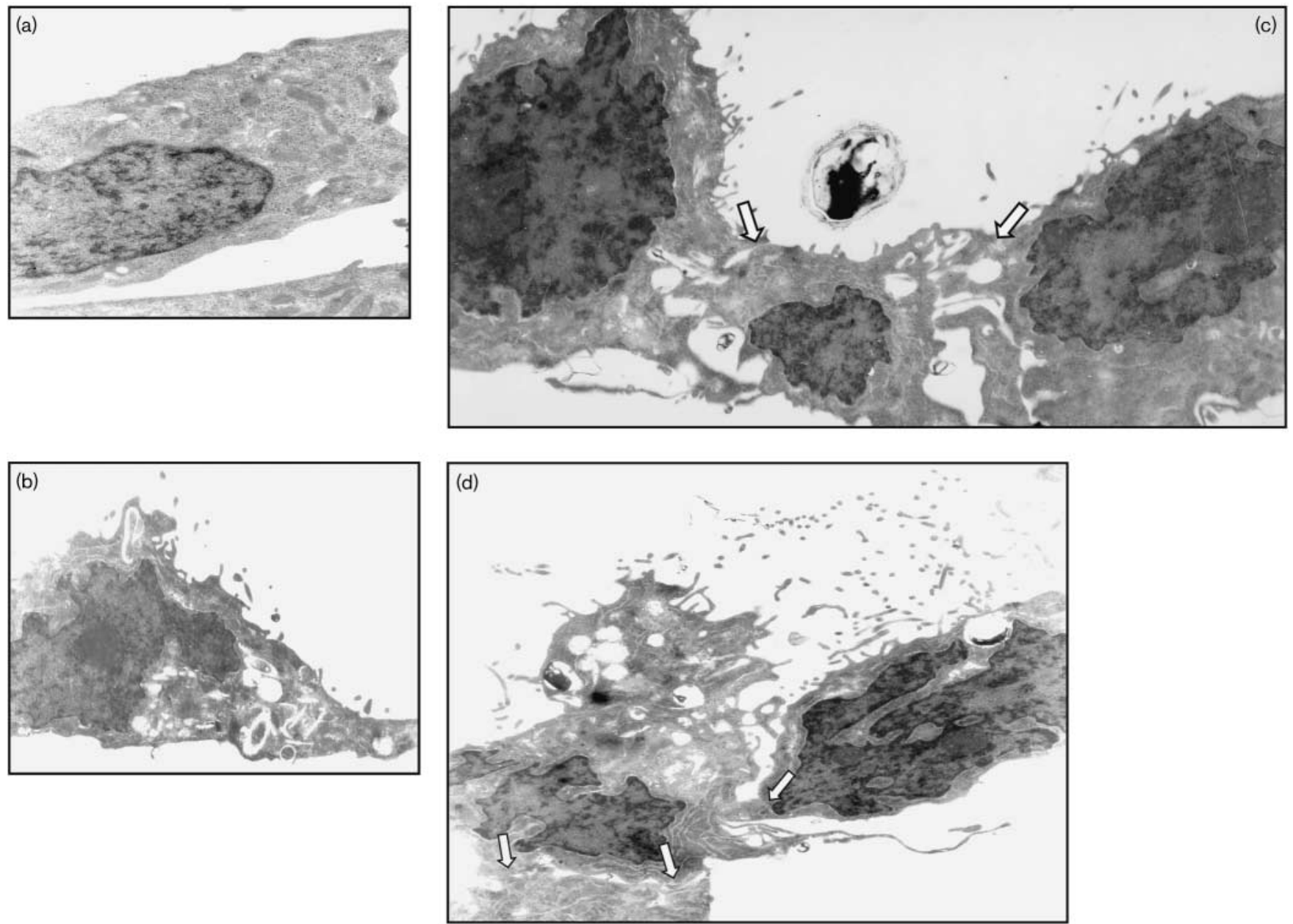

Fig. 3. Effects of $C$. neoformans on the ultrastructural morphology of HBMEC. (a) Cross-section of the HBMEC monolayer shows the morphology of HBMEC without pathogens. (b) - (d) Incubation of HBMEC with Cryptococcus cells for $4 \mathrm{~h}$ (b), $8 \mathrm{~h}$ (c) and $16 \mathrm{~h}$ (d) led to dramatic ultrastructural changes. HBMEC show a rounded morphology and ruffled membranes on the apical side and a flat appearance on the basal side, attached to the slide. Intracellular organelles show dilation of membranes. A yeast cell (cap59) is visible in the middle of panel (c). Despite the intracellular changes induced in HBMEC by Cryptococcus cells, HBMEC appear to be attached to each other (c, d; arrows).

concurrent with more drastic morphological changes and many more microvilli formations (upper right corner in Fig. 3d). In summary, Cryptococcus cells altered the morphology of HBMEC, which possibly induced cytoskeleton rearrangement.

\section{Phosphorylation regulation of cofilin in HBMEC during Cryptococcus invasion}

There is increasing evidence that cytoskeletal rearrangements, such as actin reorganization, play an essential role in pathogenic entry into host cells (Galan \& Zhou, 2000; Masuda et al., 2000; Chen et al., 2002; Eugene et al., 2002; Khan et al., 2002). To provide support for our TEM observations at the molecular level, we investigated the phosphorylation status of cofilin during Cryptococcus invasion. HBMEC extracts were prepared at different times after incubation with Cryptococcus. The samples were probed with either cofilin antibody to detect total cofilin or the specific anti-phosphorylated form of cofilin. As shown in Fig. 4 (upper panel), total cofilin was maintained at a rather constant level. However, p-cofilin was detected up to $12 \mathrm{~h}$ but began to diminish after $16 \mathrm{~h}$ (Fig. 4, lower panel). Since total cofilin did not change, the decrease in p-cofilin is most likely due to changes in phosphorylation regulation, not changes in protein expression. Thus, dephosphorylation of p-cofilin favoured cytoskeletal rearrangement during Cryptococcus invasion. Our TEM results also demonstrated marked morphological changes after prolonged incubation of HBMEC with Cryptococcus cells (Fig. 3c, d).

\section{Correlation of levels of p-cofilin with treatment of Cryptococcus with Y27632 and vanadate}

p-Cofilin was examined to analyse the effect of various treatments on the interaction of Cryptococcus with HBMEC. 


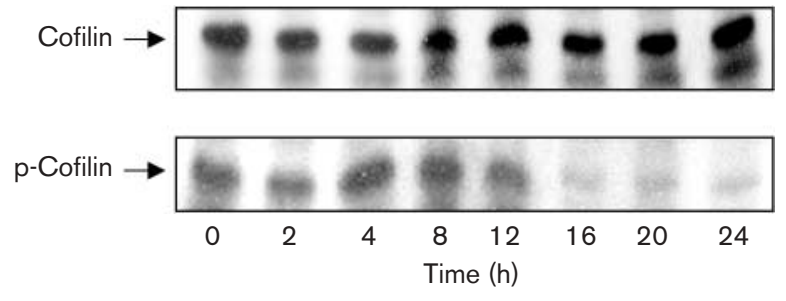

Fig. 4. Phosphorylation regulation of cofilin in HBMEC during Cryptococcus invasion. HBMEC extracts were prepared at different times after incubation with Cryptococcus cells. An equal amount of protein was loaded in each lane. Protein blots were performed using antibodies to detect total cofilin (upper panel) and p-cofilin (lower panel) using the same extracts.

HBMEC extracts were prepared after incubation with (or without) Cryptococcus cells and probed with specific antip-cofilin antibodies. In Fig. 5(a), the levels of p-cofilin, under different treatments, are shown in the upper panel and the experimental conditions are indicated underneath. In all cases, p-cofilin decreased in HBMEC incubated with C. neoformans, and, thus, it favoured increased cytoskeletal
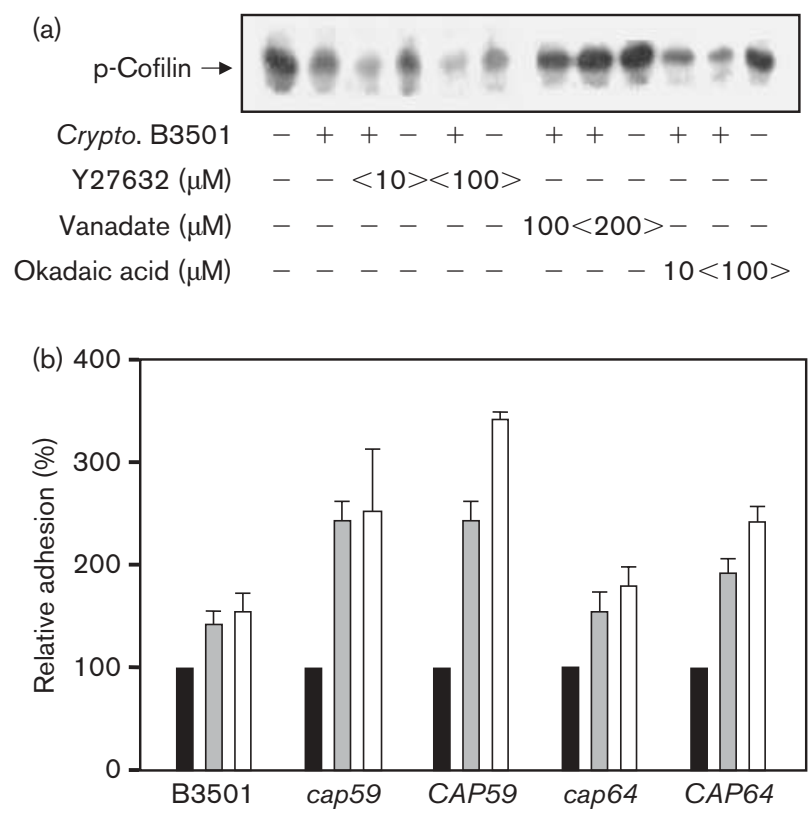

Fig. 5. Correlation of $p$-cofilin levels with various treatments and relationship to adhesion efficiency. (a) Anti-p-cofilin antibody was used to detect the phosphorylated status of $p$-cofilin by protein blotting. HBMEC extracts were prepared in the absence $(-)$ or presence (+) of $10^{6}$ Cryptococcus B3501 cells after 15 h incubation as indicated (Crypto. B3501). In the same set of experiments, some HBMEC samples were preincubated with 10 or $100 \mu \mathrm{M}$ Y27632, 100 or $200 \mu \mathrm{M}$ vanadate or 10 or $100 \mu \mathrm{M}$ okadaic acid for $1 \mathrm{~h}$ prior to extract preparation as shown. (b) Relative adhesion activity of different Cryptococcus strains in the presence of 0 (filled bars), $10 \mu \mathrm{M}$ (shaded bars) or $50 \mu \mathrm{M}$ (open bars) Y27632. Adhesion of Cryptococcus cells without Y27632 treatment was set as 100\% in individual strains. reorganization. As outlined above, the maintenance of total cofilin indicates that this reflects phosphorylation regulation, not changes in protein expression.

Rho kinase (ROCK), which is a downstream effector of Rho, does not phosphorylate cofilin directly but it phosphorylates LIMK, which, in turn, converts cofilin to the phosphorylated form, the inactive cofilin (Maekawa et al., 1999; Nakano et al., 1999). A ROCK-specific inhibitor, Y27632, was used to test this possible signalling pathway during cryptococcal invasion. In Fig. 5(a), p-cofilin was decreased by treatment with Y27632. There was an additional effect on dephosphorylation of p-cofilin with Y27632 and C. neoformans. This suggested that there was a functional link between ROCK and cofilin in HBMEC incubated with C. neoformans. The results indicated that phosphorylation of LIMK by ROCK and, consequently, increased phosphorylation of cofilin by LIMK may contribute to some of the actin cytoskeleton reorganization observed in HBMEC. This effect can be blocked by the ROCK inhibitor Y27632.

Sodium orthovanadate (inhibitor of protein tyrosine phosphatase) and okadaic acid (inhibitor of protein phosphatase 1 and phosphatase 2A) were also used in the experiment to examine their effect on the level of p-cofilin (Fig. 5a). Sodium orthovanadate increased p-cofilin, but okadaic acid showed no effect. Thus, Y27632 and vanadate function contrary to each other in terms of regulating the phosphorylation status of cofilin.

To explore the effect of Y27632 on Cryptococcus invasion, an adhesion assay was performed. Different encapsulated and acapsulated strains were tested to examine the effect of Y27632 on HBMEC (Fig. 5b). The control, without Y27632 treatment, was assigned as $100 \%$ for individual Cryptococcus strains. A titrated amount of Y27632 was added to examine the consequences of the treatments. In Fig. 5(b), it was clear that Y27632 had a similar effect among tested strains on the adhesion of HBMEC, i.e. Y27632 inhibited ROCK activity, resulting in blocking LIMK activation. The lack of LIMK activity favoured unphosphorylated cofilin and actin depolymerization activity and, thus, increasing cryptococcal adherence to HBMEC. To test the effect of vanadate on Cryptococcus invasion further, an adhesion assay was performed. Consistent with the phosphorylation status of cofilin, vanadate worked in the opposite direction, decreasing cryptococcal adherence to HBMEC from $100 \%$ to 80, $\sim 50$ and $\sim 45 \%$ with 10,100 and $200 \mu \mathrm{M}$ vanadate. Taken together, there is an intimate correlation between the phosphorylation status of cofilin and Cryptococcus adhesion to HBMEC.

\section{Confocal microscopic studies of cytoskeleton changes in HBMEC stained by phalloidin - rhodamine}

Actin filaments can be stained and observed directly by using phalloidin-rhodamine. This is useful in monitoring the organization of actin in HBMEC. In the absence of C. neoformans, actin filament bundles (or stress filaments) 
can be observed easily in HBMEC. They are normally distributed throughout the internal compartment of the cytoplasm (Fig. 6a). In the presence of C. neoformans, HBMEC has an actin cytoskeleton characterized by a marked reduction in stress fibres and a loss of straight and directed alignment of these fibres. Some denser actin aggregates become visible inside the cells, and parts of actin filaments translocate to the margin of the HBMEC. A long protrusion of actin filament staining in HBMEC can often be observed (Fig. 6b, arrow). Cryptococcus cells were not stained in this experiment. They were washed away during the sample preparation step. It was clear that the distribution of actin filaments appeared to differ in the absence and presence of Cryptococcus cells. When the phosphatase inhibitor vanadate was added, the actin filaments showed a dense patch, or dotted-shapes, inside the HBMEC (Fig. 6c). Vanadate blocked phosphatase activity, presumably favouring the phosphorylated form of cofilin, as shown in Fig. 5(a). In the presence of $C$. neoformans, the dotted actin spots were no longer observed. Instead, actin filaments located around the periphery of the membrane and some membrane extrusions were observed (Fig. 6d, arrow). It seems that C. neoformans can partially reverse the effect of vanadate. In summary, C. neoformans induces actin filament rearrangement and exerts its function(s) in a manner opposite to vanadate with regard to actin filament reorganization.

\section{Effect of Cryptococcus cells on solubility of HBMEC occludin}

Many pathogens disrupt tight junctions during invasion through activation of cytoskeletal components (Sears, 2000; Simonovic et al., 2001). Occludin is a major transmembrane protein that localizes at the tight junction. In order to determine whether there was any effect of Cryptococcus invasion during actin reorganization in HBMEC, we examined this tight junction marker, occludin, in the Triton- extractable and -insoluble fractions from HBMEC. HBMEC incubated with $C$. neoformans were harvested at different time-intervals and fractionated into Triton-insoluble and Triton-extractable fractions. The experimental conditions were similar to those used in the experiments shown in Fig. 4, except that anti-occludin antibodies were used with Tritonextractable and -insoluble fractions. Although slight differences occur in different cell types, insoluble occludin usually indicates an intact tight junction (Wachtel et al., 1999). However, once the tight junction is weakened, occludin relocates to the Triton-extractable fraction in cells. As shown in Fig. 7 (upper panel), insoluble occludin could be observed for up to $8 \mathrm{~h}$. However, it then disappeared rapidly. Some intact occludin was observed in the Triton-extractable fraction throughout the incubation (Fig. 7, lower panel). However, the degraded form became prominent after $16 \mathrm{~h}$ incubation with Cryptococcus cells, even though the TEER maintained rather constant (Fig. 2) and HBMEC still

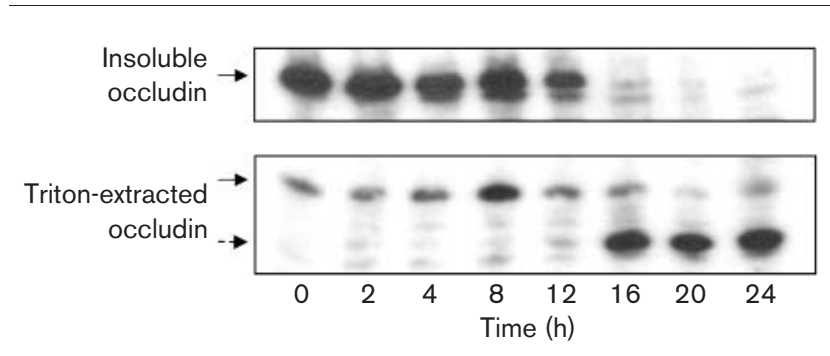

Fig. 7. Effects of Cryptococcus cells on solubility of HBMEC occludin. HBMEC were incubated with Cryptococcus B3501 and harvested at different times. HBMEC extracts were fractionated into Tritoninsoluble and Triton-extractable fractions. Protein blots were performed using anti-occludin antibodies to detect the protein in these two fractions. Solid arrows indicate occludin and the dotted arrow indicates the degraded form.
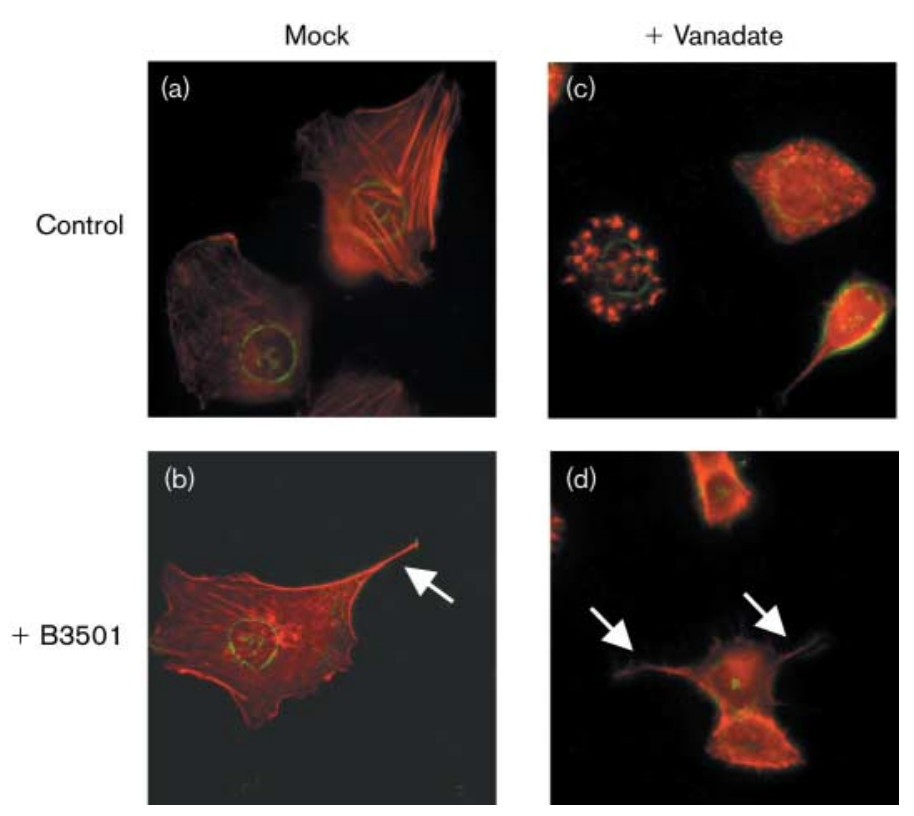

Fig. 6. Confocal microscopic studies of cytoskeletal changes of HBMEC stained with phalloidin-rhodamine to show actin filaments. Controls without Cryptococcus B3501 cells ( $a, c)$ and HBMEC preincubated with Cryptococcus cells for $2 \mathrm{~h}$ before phalloidin-rhodamine staining ( $b$, d) in the absence $(a, b)$ or presence $(c, d)$ of sodium vanadate are shown. 
associated with each other (Fig. 3). The results suggested that C. neoformans might have a profound impact on HBMEC tight junctions.

\section{DISCUSSION}

Meningitis is commonly observed in human Cryptococcus infections, implying that this micro-organism is able to breach the BBB. Despite intensive studies on the virulence factors of Cryptococcus, the underlying mechanisms by which this organism invades the CNS and causes meningitis are unclear. Since the microvascular endothelium is the major site of the $\mathrm{BBB}$, it is likely that brain microvascular endothelial cells are the primary targets for Cryptococcus cells to cross into the CNS, which results in meningeal infections. We therefore used our in vitro model of the BBB to explore the interactions between $C$. neoformans and HBMEC. This in vitro model of the BBB has been used to study the adhesion, invasion and transcytosis of bacterial pathogens across HBMEC, e.g. Escherichia coli (Huang et al., 1995, 2001; Chen et al., 2002) and Citrobacter (Badger et al., 1999), and another pathogenic yeast, Candida albicans (Jong et al., 2001). This in vitro BBB model system is particularly suitable for $C$. neoformans studies, as the pathogen has a predilection for the brain, resulting in life-threatening meningitis. Using a combination of cell adhesion and transcytosis assays (Figs 1 and 2), we showed for the first time that pathogenic C. neoformans is able to bind to, and transcytose across, HBMEC monolayers. The capsule is a major virulence factor of $C$. neoformans. Our adhesion assay showed some differences between isogenic capsulated and acapsulated strains in the adhesion assay (Fig. 1). However, in the transcytosis studies, the isogenic pairs CAP64 vs cap64 and CAP59 vs cap59 showed somewhat different efficiencies in crossing the HBMEC monolayer (Fig. 2). The acapsular cap64 and cap59 cells have a tendency to aggregate together. The clumpy nature of cap mutants may make them less adherent and less mobile. Interestingly, the virulent strain B3501, which is heavily capsulated, has the least adhesion ability. One explanation is that B3501 cells are too bulky to cross the HBMEC monolayer effectively. Though we cannot rule out other possibilities, it seems that some of the physical properties (size, clumping, etc.) of the capsular components may affect their adhesion and invasion abilities in cell cultures.

One striking observation is that HBMEC undergo considerable morphological alterations during Cryptococcus infection. The membrane ruffling induced by Cryptococcus resembles the changes in the plasma membrane induced by growth factors and oncogenic stimuli (Bar-Sagi \& Feramisco, 1986). This effect has been observed in other eukaryotic cells with several pathogens, such as Salmonella and Chlamydia (Wyrick et al., 1989; Francis et al., 1993). These observations indicate that actin cytoskeleton reorganization may occur in HBMEC in response to cryptococcal invasion. The rapid turnover of actin filaments and the tertiary meshwork formation are regulated by a variety of actin-binding proteins. Phosphorylation regulation of cofilin plays a definitive role in this process (Arber et al., 1998; Yang et al., 1998; Sumi et al., 1999). Our data demonstrate the relationships among cofilin phosphorylation, actin reorganization and the ability of cryptococci to adhere to HBMEC (Figs 4 and 5). The results also strongly suggest an involvement of the ROCK $\rightarrow$ LIMK $\rightarrow$ cofilin pathway (Maekawa et al., 1999; Nakano et al., 1999). However, the upstream signal is unclear, and the downstream effects are expected to be complicated. For example, the LIMK family has two members, LIMK-1 and LIMK-2 (Nunoue et al., 1995). Their functions are under the control of distinct Rho subfamily GTPases: LIMK-1 is regulated by Rac, relaying a signal to the formation of lamellipodia, and LIMK-2 is activated through Rho and Cdc42, participating in the formation of stress fibres, focal adhesion and filopodia (Sumi et al., 2001). It is further complicated by the fact that LIMK can be regulated by another kinase, PAK, in addition to ROCK (Edwards et al., 1999; Dan et al., 2001). ROCK may regulate occludin function, branching its effects on the permeability of the tight junction (Hirase et al., 2001). Other signalling pathways are also possible. For example, ROCK has an essential role in phosphorylation of myosin light chain, leading to membrane blebbing and morphological changes in several cell lines (Song et al., 2002). Many of the signalling regulations are tissue- or cell type-specific. Intertwining signals may also exist in HBMEC to govern such complicated morphological changes. Our studies provide a starting point for many future studies of cryptococcal traversal across the BBB.

It has previously been shown that Candida albicans is able to invade and multiply in HBMEC (Jong et al., 2001). Candida was present in host cytoplasm in large vacuoles and subsequently exits from the basolateral side of the cells without affecting monolayer integrity, a possible transcellular mechanism of crossing the BBB (Huang \& Jong, 2001). Such a mechanism was not evident for Cryptococcus. In addition, the efficiency of adhesion and invasion of Cryptococcus is much lower, by about one order of magnitude, than that of Candida albicans. Nevertheless, both fungal pathogens can pass through HBMEC monolayers, though with different efficiencies (Fig. 2). It is currently unknown whether Candida albicans affects cofilin regulation and occludin redistribution, as $C$. neoformans does. Another difference between the two fungal pathogens is that Candida albicans can be observed easily inside HBMEC by TEM. However, we are unable to demonstrate clearly, at this time, any intracellular C. neoformans cells after extensive TEM studies. Instead, Cryptococcus induced considerable morphological changes, for example the appearance of irregular nuclei, swollen organelles and membrane ruffling (Fig. 3). Membrane ruffling may facilitate ingestion of the pathogen (Wyrick et al., 1989; Francis et al., 1993) or alteration of tight junction permeability (Matter \& Balda, 1999; Tsukita et al., 1999). There may be other functions that will become apparent during our studies. It has been reported previously that HUVEC can phagocytose C. neoformans cells (Ibrahim et al., 1995). However, BBB endothelial cells have an extremely low 
rate of endocytosis vesicles and represent a restrictive paracellular diffusion barrier. It is plausible that $C$. neoformans traverses across HUVEC and HBMEC by different mechanisms.

Occludin is a major transmembrane protein in endothelial cells (Hirase et al., 1997). The protein can be regulated by RhoA-ROCK-dependent and -independent mechanisms (Hirase et al., 2001). Current data indicate that occludin plays a regulatory rather than structural role in tight junctions (Kuwabara et al., 2001). It has been shown that the proteolysis of occludin is associated with enhanced paracellular permeability and redistribution of the molecule from sites of cell contact in HUVEC (Wachtel et al., 1999). Alterations to occludin were observed in HBMEC incubated with C. neoformans, raising the possibility that Cryptococcus may cross the BBB through a paracellular pathway. More studies are necessary to clarify this mechanism. Other possibilities exist: for example, the Cryptococcus cells could be engulfed by immune cells and carried through the barrier (a 'Trojan horse' mechanism) (Huang \& Jong, 2001) or, perhaps, could breach the HBMEC monolayer by the induction of apoptosis in host cells. Crossing of the BBB may be achieved by a combination of mechanisms. The mechanisms of Cryptococcus traversal across the BBB monolayer require further investigation. We are currently isolating active components from Cryptococcus cells to unravel the cell wall components that induce membrane ruffling and morphological changes in HBMEC.

\section{ACKNOWLEDGEMENTS}

We wish to thank Drs Albert S. Lossinksy and Chao-Hung Lee for their critical comments. We also thank George McNamara for operating the CHLA Image Core confocal microscope. This research was supported by a grant to the Neil Bogart Memorial Laboratories by the T. J. Martell Foundation (A. Y. J.), by the American Heart Association 0150094N (A. Y. J.) and by NIH grants R29 AI40635 (S.-H. H.), RO1 NS26310, AI47225 and HL61951 (K. S. K.).

\section{REFERENCES}

Arber, S., Barbayannis, F. A., Hanser, H., Schneider, C., Stanyon, C. A., Bernard, O. \& Caroni, P. (1998). Regulation of actin dynamics through phosphorylation of cofilin by LIM-kinase. Nature 393, 805-809.

Badger, J. L., Stins, M. F. \& Kim, K. S. (1999). Citrobacter freundii invades and replicates in human brain microvascular endothelial cells. Infect Immun 67, 4208-4215.

Bamburg, J. R. (1999). Proteins of the ADF/cofilin family: essential regulators of actin dynamics. Annu Rev Cell Dev Biol 15, 185-230.

Bar-Sagi, D. \& Feramisco, J. R. (1986). Induction of membrane ruffling and fluid-phase pinocytosis in quiescent fibroblasts by ras proteins. Science 233, 1061-1068.

Bierne, H., Gouin, E., Roux, P., Caroni, P., Yin, H. L. \& Cossart, P. (2001). A role for cofilin and LIM kinase in Listeria-induced phagocytosis. J Cell Biol 155, 101-112.

Broadwell, R. D., Baker-Cairns, B. J., Friden, P. M., Oliver, C. \& Villegas, J. C. (1996). Transcytosis of protein through the mammalian cerebral epithelium and endothelium. III. Receptor-mediated transcytosis through the blood-brain barrier of blood-borne transferrin and antibody against the transferrin receptor. Exp Neurol 142, 47-65.
Buchanan, K. L. \& Murphy, J. W. (1998). What makes Cryptococcus neoformans a pathogen? Emerg Infect Dis 4, 71-83.

Chang, Y. C. \& Kwon-Chung, K. J. (1994). Complementation of a capsule-deficient mutation of Cryptococcus neoformans restores its virulence. Mol Cell Biol 14, 4912-4919.

Chang, Y. C. \& Kwon-Chung, K. J. (1998). Isolation of the third capsuleassociated gene, CAP60, required for virulence in Cryptococcus neoformans. Infect Immun 66, 2230-2236.

Chang, Y. C. \& Kwon-Chung, K. J. (1999). Isolation, characterization, and localization of a capsule-associated gene, CAP10, of Cryptococcus neoformans. J Bacteriol 181, 5636-5643.

Chang, Y. C., Penoyer, L. A. \& Kwon-Chung, K. J. (1996). The second capsule gene of Cryptococcus neoformans, CAP64, is essential for virulence. Infect Immun 64, 1977-1983.

Chen, Y. H., Chen, S. H., Jong, A., Zhou, Z. Y., Li, W., Suzuki, K. \& Huang, S. H. (2002). Enhanced Escherichia coli invasion of human brain microvascular endothelial cells is associated with alternations in cytoskeleton induced by nicotine. Cell Microbiol 4, 503-514.

Dan, C., Kelly, A., Bernard, O. \& Minden, A. (2001). Cytoskeletal changes regulated by the PAK4 serine/threonine kinase are mediated by LIM kinase 1 and cofilin. J Biol Chem 276, 32115-32121.

Edwards, D. C., Sanders, L. C., Bokoch, G. M. \& Gill, G. N. (1999). Activation of LIM-kinase by Pak1 couples Rac/Cdc42 GTPase signalling to actin cytoskeletal dynamics. Nat Cell Biol 1, 253-259.

Eugene, E., Hoffmann, I., Pujol, C., Couraud, P. O., Bourdoulous, S. \& Nassif, X. (2002). Microvilli-like structures are associated with the internalization of virulent capsulated Neisseria meningitidis into vascular endothelial cells. J Cell Sci 115, 1231-1241.

Feldmesser, M., Kress, Y., Novikoff, P. \& Casadevall, A. (2000). Cryptococcus neoformans is a facultative intracellular pathogen in murine pulmonary infection. Infect Immun 68, 4225-4237.

Francis, C. L., Ryan, T. A., Jones, B. D., Smith, S. J. \& Falkow, S. (1993). Ruffles induced by Salmonella and other stimuli direct macropinocytosis of bacteria. Nature 364, 639-642.

Galan, J. E. \& Zhou, D. (2000). Striking a balance: modulation of the actin cytoskeleton by Salmonella. Proc Natl Acad Sci U S A 97, 8754-8761.

Gottfredsson, M. \& Perfect, J. R. (2000). Fungal meningitis. Semin Neurol 20, 307-322.

Hamilton, A. J. \& Goodley, J. (1996). Virulence factors of Cryptococcus neoformans. Curr Top Med Mycol 7, 19-42.

Hirase, T., Staddon, J. M., Saitou, M., Ando-Akatsuka, Y., Itoh, M., Furuse, M., Fujimoto, K., Tsukita, S. \& Rubin, L. L. (1997). Occludin as a possible determinant of tight junction permeability in endothelial cells. J Cell Sci 110, 1603-1613.

Hirase, T., Kawashima, S., Wong, E. Y., Ueyama, T., Rikitake, Y., Tsukita, S., Yokoyama, M. \& Staddon, J. M. (2001). Regulation of tight junction permeability and occludin phosphorylation by Rhoap160ROCK-dependent and -independent mechanisms. J Biol Chem 276, 10423-10431.

Hogan, L. H., Klein, B. S. \& Levitz, S. M. (1996). Virulence factors of medically important fungi. Clin Microbiol Rev 9, 469-488.

Huang, S. H. \& Jong, A. Y. (2001). Cellular mechanisms of microbial proteins contributing to invasion of the blood-brain barrier. Cell Microbiol 3, 277-287.

Huang, S. H., Wass, C., Fu, Q., Prasadarao, N. V., Stins, M. \& Kim, K. S. (1995). Escherichia coli invasion of brain microvascular endothelial cells in vitro and in vivo: molecular cloning and characterization of invasion gene ibe10. Infect Immun 63, 4470-4475.

Huang, S. H., Wan, Z. S., Chen, Y. H., Jong, A. Y. \& Kim, K. S. (2001). 
Further characterization of Escherichia coli brain microvascular endothelial cell invasion gene ibeA by deletion, complementation, and protein expression. J Infect Dis 183, 1071-1078.

Ibrahim, A. S., Filler, S. G., Alcouloumre, M. S., Kozel, T. R., Edwards, J. E., Jr \& Ghannoum, M. A. (1995). Adherence to and damage of endothelial cells by Cryptococcus neoformans in vitro: role of the capsule. Infect Immun 63, 4368-4374.

Jong, A. Y., Stins, M. F., Huang, S. H., Chen, S. H. \& Kim, K. S. (2001) Traversal of Candida albicans across human blood-brain barrier in vitro. Infect Immun 69, 4536-4544.

Khan, N. A., Wang, Y., Kim, K. J., Chung, J. W., Wass, C. A. \& Kim, K. S. (2002). Cytotoxic necrotizing factor-1 contributes to Escherichia coli K1 invasion of the central nervous system. J Biol Chem 277, 15607-15612.

Kim, K. S. (2001). Escherichia coli translocation at the blood-brain barrier. Infect Immun 69, 5217-5222.

Kuwabara, H., Kokai, Y., Kojima, T., Takakuwa, R., Mori, M. \& Sawada, N. (2001). Occludin regulates actin cytoskeleton in endothelial cells. Cell Struct Funct 26, 109-116.

Kwon-Chung, K. J. \& Rhodes, J. C. (1986). Encapsulation and melanin formation as indicators of virulence in Cryptococcus neoformans. Infect Immun 51, 218-223.

Kwon-Chung, K. J., Sorrell, T. C., Dromer, F., Fung, E. \& Levitz, S. M. (2000). Cryptococcosis: clinical and biological aspects. Med Mycol 38 (Suppl. 1), 205-213.

Maekawa, M., Ishizaki, T., Boku, S. \& 7 other authors (1999). Signaling from Rho to the actin cytoskeleton through protein kinases ROCK and LIM-kinase. Science 285, 895-898.

Masuda, M., Betancourt, L., Matsuzawa, T., Kashimoto, T., Takao, T., Shimonishi, Y. \& Horiguchi, Y. (2000). Activation of rho through a cross-link with polyamines catalyzed by Bordetella dermonecrotizing toxin. EMBO J 19, 521-530.

Matter, K. \& Balda, M. S. (1999). Occludin and the functions of tight junctions. Int Rev Cytol 186, 117-146.

Mitchell, T. G. \& Perfect, J. R. (1995). Cryptococcosis in the era of AIDS - 100 years after the discovery of Cryptococcus neoformans. Clin Microbiol Rev 8, 515-548.

Nakano, K., Takaishi, K., Kodama, A., Mammoto, A., Shiozaki, H., Monden, M. \& Takai, Y. (1999). Distinct actions and cooperative roles of ROCK and mDia in Rho small $\mathrm{G}$ protein-induced reorganization of the actin cytoskeleton in Madin-Darby canine kidney cells. Mol Biol Cell 10, 2481-2491.

Niwa, R., Nagata-Ohashi, K., Takeichi, M., Mizuno, K. \& Uemura, T. (2002). Control of actin reorganization by Slingshot, a family of phosphatases that dephosphorylate ADF/cofilin. Cell 108, 233-246.

Nizet, V., Kim, K. S., Stins, M., Jonas, M., Chi, E. Y., Nguyen, D. \& Rubens, C. E. (1997). Invasion of brain microvascular endothelial cells by group B streptococci. Infect Immun 65, 5074-5081.
Nunoue, K., Ohashi, K., Okano, I. \& Mizuno, K. (1995). LIMK-1 and LIMK-2, two members of a LIM motif-containing protein kinase family. Oncogene 11, 701-710.

Perfect, J. R., Wong, B., Chang, Y. C., Kwon-Chung, K. J. \& Williamson, P. R. (1998). Cryptococcus neoformans: virulence and host defences. Med Mycol 36 (Suppl. 1), 79-86.

Rubin, L. L. \& Staddon, J. M. (1999). The cell biology of the blood-brain barrier. Annu Rev Neurosci 22, 11-28.

Sears, C. L. (2000). Molecular physiology and pathophysiology of tight junctions. V. Assault of the tight junction by enteric pathogens. Am J Physiol Gastrointest Liver Physiol 279, G1129-G1134.

Simonovic, I., Arpin, M., Koutsouris, A., Falk-Krzesinski, H. J. \& Hecht, G. (2001). Enteropathogenic Escherichia coli activates ezrin, which participates in disruption of tight junction barrier function. Infect Immun 69, 5679-5688.

Song, Y., Hoang, B. Q. \& Chang, D. D. (2002). ROCK-II-induced membrane blebbing and chromatin condensation require actin cytoskeleton. Exp Cell Res 278, 45-52.

Stins, M. F., Gilles, F. \& Kim, K. S. (1997a). Selective expression of adhesion molecules on human brain microvascular endothelial cells. J Neuroimmunol 76, 81-90.

Stins, M. F., Prasadarao, N. V., Zhou, J., Arditi, M. \& Kim, K. S. (1997b). Bovine brain microvascular endothelial cells transfected with SV40large $\mathrm{T}$ antigen: development of an immortalized cell line to study pathophysiology of CNS disease. In Vitro Cell Dev Biol Anim 33, 243-247.

Sumi, T., Matsumoto, K., Takai, Y. \& Nakamura, T. (1999). Cofilin phosphorylation and actin cytoskeletal dynamics regulated by rho- and Cdc42-activated LIM-kinase 2. J Cell Biol 147, 1519-1532.

Sumi, T., Matsumoto, K. \& Nakamura, T. (2001). Specific activation of LIM kinase 2 via phosphorylation of threonine 505 by ROCK, a Rhodependent protein kinase. J Biol Chem 276, 670-676.

Tsukita, S., Furuse, M. \& Itoh, M. (1999). Structural and signalling molecules come together at tight junctions. Curr Opin Cell Biol 11, 628-633.

Wachtel, M., Frei, K., Ehler, E., Fontana, A., Winterhalter, K. \& Gloor, S. M. (1999). Occludin proteolysis and increased permeability in endothelial cells through tyrosine phosphatase inhibition. J Cell Sci 112, 4347-4356.

Wyrick, P. B., Choong, J., Davis, C. H., Knight, S. T., Royal, M. O., Maslow, A. S. \& Bagnell, C. R. (1989). Entry of genital Chlamydia trachomatis into polarized human epithelial cells. Infect Immun 57, 2378-2389.

Yang, N., Higuchi, O., Ohashi, K., Nagata, K., Wada, A., Kangawa, K. Nishida, E. \& Mizuno, K. (1998). Cofilin phosphorylation by LIMkinase 1 and its role in Rac-mediated actin reorganization. Nature 393, 809-812. 